\title{
PROGRESS IN A MASONRY TASKING ROBOT
}

\author{
Denis Chamberlain, Philip speare and seshagiri Ala \\ Construction Robotics Unit, structures Research Centre \\ City University, Northampton square \\ London, EClv OHB, England
}

\begin{abstract}
Four major strands of development are reported: the experimental robot cell for masonry tasking currently being built is described, the operational tolerances required are established and the means of achieving them discussed, an account is given of work undertaken on the production, dispensing and application of bond materials and a CAD model for vision sensing for use in design and construction planning is outlined. Although the initial development is concerned with the robotisation of masonry tasks, the techniques being developed will have wider applications in relation to inspection and repair.
\end{abstract}

\section{INTRODUCTION}

The total annual labour element in brickwork and blockwork construction in the UK has a substantial value[1]. This and factors such as the increasing skill shortage has prompted research into the automation and robotisation of masonry tasks. In this, the distinction is made between essentially 'hard logic' automation machines and advanced 'soft logic' robot equipment operating under closed loop control regimes. This paper relates progress in the development of enabling technology for the latter.

Figure 1 identifies the various elements of the experimental robot cell currently being built. The functions of the conveyor are to survey and deliver only suitable block material to the gripper presentation point. Survey covers sizing and evaluation of geometric defects which must comply with quality requirements. Size tolerances are accepted in the supply material as this will be cheaper to manufacture than precise material. The function of the combined mixer pump unit is to deliver a standard consistency bond $\operatorname{mix}$ to the bonding surfaces. A special nozzle has been developed which issues the mix to a set profile for in situ or pre-insertion application. A gantry robot 2 f with both mechanical and absorption gripper option has been adopted to perform the main manipulation tasks with navigational assistance from the rising laser planer. Various types of sensors are employed in the cell, vision sensing being a major element.

In what follows, the robot control requirement will be seen to arise from consideration of the strength requirement in finished work. Control and operation of the cell is discussed and observations made on the practicalities of delivering suitable bond material. The final section outlines developments in the CAD based vision strategy. 
It is anticipated that the cell assembly will be completed by late July 1991, followed by a series of experimental constructions. Other projects relating to inspection and repair of masonry and reinforced concrete structures are also envisaged.

strength reducing factors in wall construction include furrowed mortar bedding, overthick mortar bed, plan bow and outof-plumb. These are work quality factors rather material which is considered later. A further factor not considered here is creep which is known to reduce both shear and compressive strength. Other facets of strength are flexural bond, flexural tension and direct tension actions, their relative importance being dependent on the functions of the masonry element.

Furrowed bedding amounts to a reduction in the area of the bond interface, and arises from a combination of insufficient mortar and poor application method. As an individual defect, mortar and poor application ming a
furrowed bedding could reduce compressive strength by $24 \%[3]$. A
similar defect, though generally considered to relate to serviceability rather than strength, is imperfect perpend jointing (vertical fill between block units).

In UK the standard mortar bed thickness is $10 \mathrm{~mm}$ and reduction of this to practical limits results in an increase in the wall strength. However, thicker bedding results in strength reduction depending on the block and mortar strengths. This reduction increases with bed thickness, brick strength, and by implication, mortar strength, though some anomalies are apparent in test results. Considering all possibilities, a bed thickness of $14 \mathrm{~mm}$ should not lead to compressive or shear strength reductions greater than 12\%. Whilst tests are somewhat inconclusive, beds from $16 \mathrm{~mm}-20 \mathrm{~mm}$ could broviding expected to produce $30 \%$ reduction in compressive strength. Providing the bed application is flat with thickness controlled to $\pm 3 \mathrm{~mm}$, strength application is flat with thick covered thus far are contained. Even if all blocks were
fandersized or oversized, this margin could be absorbed in the bedding down process.

Plan bow and out-of-plumb are geometrical defects of the same type, both equivalent to the eccentric application of loading. For a $20 \mathrm{~mm}$ out-of-plan defect, tests have shown the compressive strength can be reduced by about $15 \%$ and shear strength by $18 \%$ for a $102.5 \mathrm{~mm}$ thick wall construction. The relative thickness of the construction and the effective aspect ratio are decisive in this matter, with theoretical reduction tending to be higher than those determined in tests. An out of plane tolerance of $\pm 6 \mathrm{~mm}$ would seem to cover this factor adequately, though detailed calculations should be applied to each construction project. 
In the context of manufacturing robots, the tolerances indicated are generous. However, in the context of a large envelope mobile construction robot the material application and positioning requirements merit careful consideration.

\section{ROBOT CELI ELEMENTS}

\section{Material supply}

Block and brick units are placed manually on the conveyor belt. In some future robotic plant development, dehacking robots could be employed in a manner similar to that in brick manufacturing. Proprietary unit size tolerances range from $\pm 1 \mathrm{~mm}$ to $\pm 4 \mathrm{~mm}$, thus it is necessary to size units at the supply stage. Damage to corners and edges is not uncommon and units must be checked for possible rejection.

A microprocessor driven conveyor is used for delivery, inspection and presentation of units, this having velocity and position control to its motor. By a combination of 'through beam' photoelectric switches and focused ultrasonic analogue position sensors, a unit is accurately located for vision processing and final presentation. Accurate positioning at the camera inspection station enables efficient CAD based vision sensing for size and damage determination. Unacceptable material is rejected and unit dimension data is transferred to the 'as built' CAD model discussed later. Communication between the host processor, conveyor and vision processors ensures the continuous delivery of suitable units.

\section{Robot And End Effectors}

The central element of the cell is an industry standard robot based on the CROCUS system. This is an electric robot having a working envelope of $2.0 \mathrm{~m}$ (vertical) $\times 4.0 \mathrm{~m} \times 2.5 \mathrm{~m}$ with $360^{\circ}$ yaw and $360^{\circ}$ pitch/roll to its end effector. With the pitch/roll revolute locked, the payload is $500 \mathrm{~kg}$ and for full manipulation the payload is $30 \mathrm{~kg}$. Whilst lower density blocks are envisaged, this corresponds to a $100 \mathrm{~mm} \times 250 \mathrm{~mm} \times 500 \mathrm{~mm}$ block of normal structural concrete. Two types of block grippers are available for the robot, a clamp with $90 \mathrm{~mm}$ - $110 \mathrm{~mm}$ working opening and a double cup absorption device. Texture plays an important in the effectiveness of these, part in this, some proprietary materials being entirely incompatible with the absorption device. Figure 2 illustrates details of the clamp device. Sensor provisions for these end effectors include 'eyein-hand' vision, ranging, clamp force, block seating, loading, impact avoidance and detection, and overload protection. An air powered multi pressure overload device (POD) has been adopted for full compliance on overload at the end effector.

\section{Robot Control}

A Smart Motor Controller Card (SMCC) has been adopted for the robot cell, this providing closed loop motion control under high level instruction from the host computer. Position, speed, acceleration and force control are achieved with this. Cards are 
daisy chained from a master SMCC which distributes processing on the basis of two axes per card. Programs can be dumped on each card and background programmable Logic controllers (PLC) are provided which allow I/O functions to be executed independently of motion commands, yet also providing I/O programming relative to position, velocity or other requirements. Key sensors and auxiliary equipment such as the conveyor and pump can thus be properly integrated. The SMCC's high speed architecture enables very fast servo update times of the order of $150 \mu$ s for simultaneous motion on two axes. Apart from a dedicated BASIClike high level command knowledge, ' $C$ ' programming is used with RS $232 \mathrm{C}$ data communications to 38400 baud.

\section{Bond Mix Design And Delivery}

In addition to the usual requirements for cohesion and In addition to the usual rise fors
adhesion, conflicting factors arise in the design of the bond
material. Whilst it must be sufficiently stiff to support blocks during the laying process, long term strength should generally be moderate in relation to the block strength. If this policy is neglected then subsequent environmental loading is likely to lead to actual block failure rather than more acceptable joint movement. Unfortunately, in the case of cementitious material, reduction in the water/solids ratio tends to elevate the long term strength as well as stiffen the consistency. Considering now that the workability necessary for pumping improves with increase in this ratio, the problem is fully stated.

For cement:sand and cement:sand:lime based mixes some admixtures are a common can considerably reduce their effectiveness. Currently,
encouraging results are being obtained with a PFA (Pulverised Fuel Ash) based mix under collaborative development with Pozament UK. It is envisaged that this would be pre-batched, requiring only the addition of a fixed quantity of water. As a volume product, costs are expected to be actually higher than for conventional materials, though pre-batching would tend to lead to savings.

Controlled delivery rates up to about 5 litres/min are required for automation, a low rate for most pumps of suitable type. Types under consideration are: (i) peristaltic, pneumatic, and (iii) auger.

Peristaltic devices are attractive because the mix is isolated from any mechanism, thus tending to prolong the useful pumping interval. Multiple cams are recommended with this type in order to reduce the surge effect. The pneumatic type has not been tested to date. A Metrix pressure pointing auger pump is currently employed in the development of the mix and delivery system, as illustrated in Figure 3. This is a two part system comprising mixer and pump, the mixer unit being mobile and therefore able to serve a number of pump stations. A pressure sensor has been installed at the pump outlet in order to detect blockages and thus allow a lighter supply line to be employed. 
Flow control is achieved by solenoid valve which regulates the power supply to the pump motor. Run times of the order of three hours are achievable with typical cleaning time of 20 minutes, using a high pressure water jet. A nozzle has been developed for mortar dispensing, which provides a well formed bed without furrows.

\section{work Referencing And Navigation}

Recognising that the point-to-point performance of a fixed origin gantry robot is out of keeping with a mobile robot's
requirement for navigation and referencing, only the resolution facility is utilised. Where appropriate, targets and completed work are used as reference objects. The main component in this is a spectra physics rotating laser providing a horizontal reference plane to $\pm 1.5 \mathrm{~mm}$ at $10 \mathrm{~m}$ radius. This is mounted on a rising platform such that the robot can request the appropriate reference level. A companion eye provides an active response to the beam which also strikes objects for vision processing. By

correlation with the CAD model, independence from the robot's own reference system is achieved.

\section{CAD MODELIING FOR VISION SENSING}

A comprehensive CAD model is being developed for design and construction planning of masonry projects, this having attributes for sensing in material inspection, manipulation and robot navigation. Two versions of the model coexist, these being 'as planned' and 'as built'. Currently the GRASP robot simulation software is used to express designs and simulate construction, with simplified sensor representation. Using a simulated camera viewpoint and hidden surface processing, scenes for vision processing and general sensor interaction with objects can be reviewed. In fact, it is in vision sensing that the CAD model representation plays a major role, providing the key to efficient implementation. For while vision sensing is unquestionably the most flexible type of sensing, the overheads are potentially prohibitive in terms of computation. This can be readily appreciated on consideration that a 512 × 512 × 8 bit/pixel frame grabber, operating at video rate, delivers some 8 Mbits of data/second.

By the CAD model representation, the object composition of a scene is known a priori as a function of viewpoint and direction. Furthermore, with complementary sensing the orientation and position of individual objects and assemblies are known to some level of certainty and proximity. The accuracy is determined by the robot accuracy and response to navigational aids. This knowledge can be used to mask off unwanted pixel regions, margins provided for anticipated zones of detail such as edges according to the accuracy requirement. Using this technique, the volume of pixels submitted for gray level processing can be reduced to a small proportion of the total scene, thus enhancing processing speed. 
A universal data structure[5] termed UDS, which aids the design of optimal boundary data structures, has led to the discovery of more efficient data structures than commonly employed. Three basic entities are set in a data unit, Vertex, Edge and Face, these augmented by five further entities which are Universe, Body, Cavity, Loop and segment. Based on this, a compact object data structure can be created with very efficient access times. Research in the area is continuing, the ultimate objective being realistic performance for both non-manipulative and manipulative operations on $C A D$ based robot work in example of a source and segmented wall scene is shown in Figure 4 .

\section{CONCLUSIONS}

A comprehensive approach to the robotisation of masonry related tasks has been described covering the development of the robot cell, the establishment material and a CAD vision system.

In respect of tolerances, it is concluded that the accuracy requirements for the robot are defined by strength related tolerances of the finished construction such as bow, out-of-plumb and thickness and evenness of the mortar bed, and by dimensional tolerances of the units.

The report of work to date on bond materials shows that a mix can be designed which allows the conflicting requirements of mix can be designed which allows the conflicting require strength
pumpability, stiffness during placing and bedding and stilly
compatibility with the blocks to be resolved.

A CAD model is outlined which is demonstrated to be the most efficient way of proceeding with respect to vision. The flexibility of vision sensing makes s can prohibit its use. The associated computational requir pixels required for processing to be minimized and hence realistic robot work scenes to be produced.

\section{ACRNOWLEDGEMENTS} The authors acknowledge with gratitude the financial
support of the Science and Engineering Research council without
6. Ackil which this work could not continue.

Thanks are also due to Metrix Engineering UK Ltd for the Thanks are also due to Metrix Engineering UK Lointing equipment, Pozament UK Ltd for
supply of pressure point
supplying bonding materials and spectra Physics UK Ltd for supplying laser equipment. 


\section{REFERENCES}

1. CHAMBERLAIN, D. A. Development In Construction Robotics At City University, Construction Automation Systems And Robotics Symposium, Reading University, 2 lst March 1991.

2. CHAMBERLAIN, D. A., SPEARE, P. R. S. AND WEST, G. A. A Masonry Tasking Robot, Mechatronic Systems Engineering, Vol. 1. pp 139-147, Kluwer Academic Publishers, 1990.

3. MUMENYA, S. W. The Effect of Geometric Imperfections on The Strength of Structural Brickwork, MSc Thesis, City
University, 1990.

4. ALA, S. R. AND CHAMBERLAIN, D. A. An Efficient Boundary Data structure For CAD And Robotics, 6th International Conference on CAD/CAM, Robotics And Factories of The Future, August, 1991 (To be Published).

5. ALA, S. R. Design Methodology of Boundary Data structures, ACM Symposium on Solid Modelling Foundations And CAD/CAM 


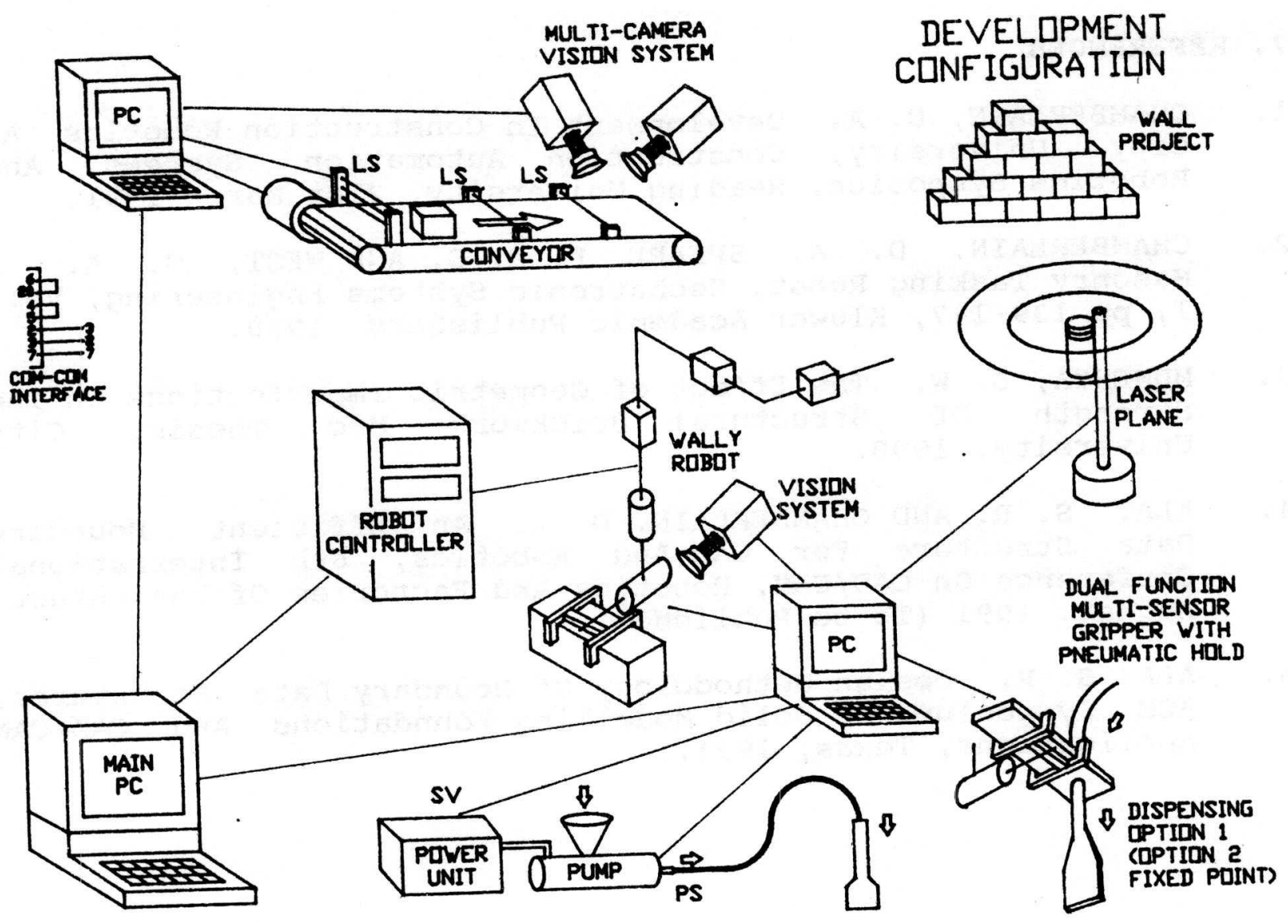

Figure 1. Experimental Robot Cell for Masonry Tasking 


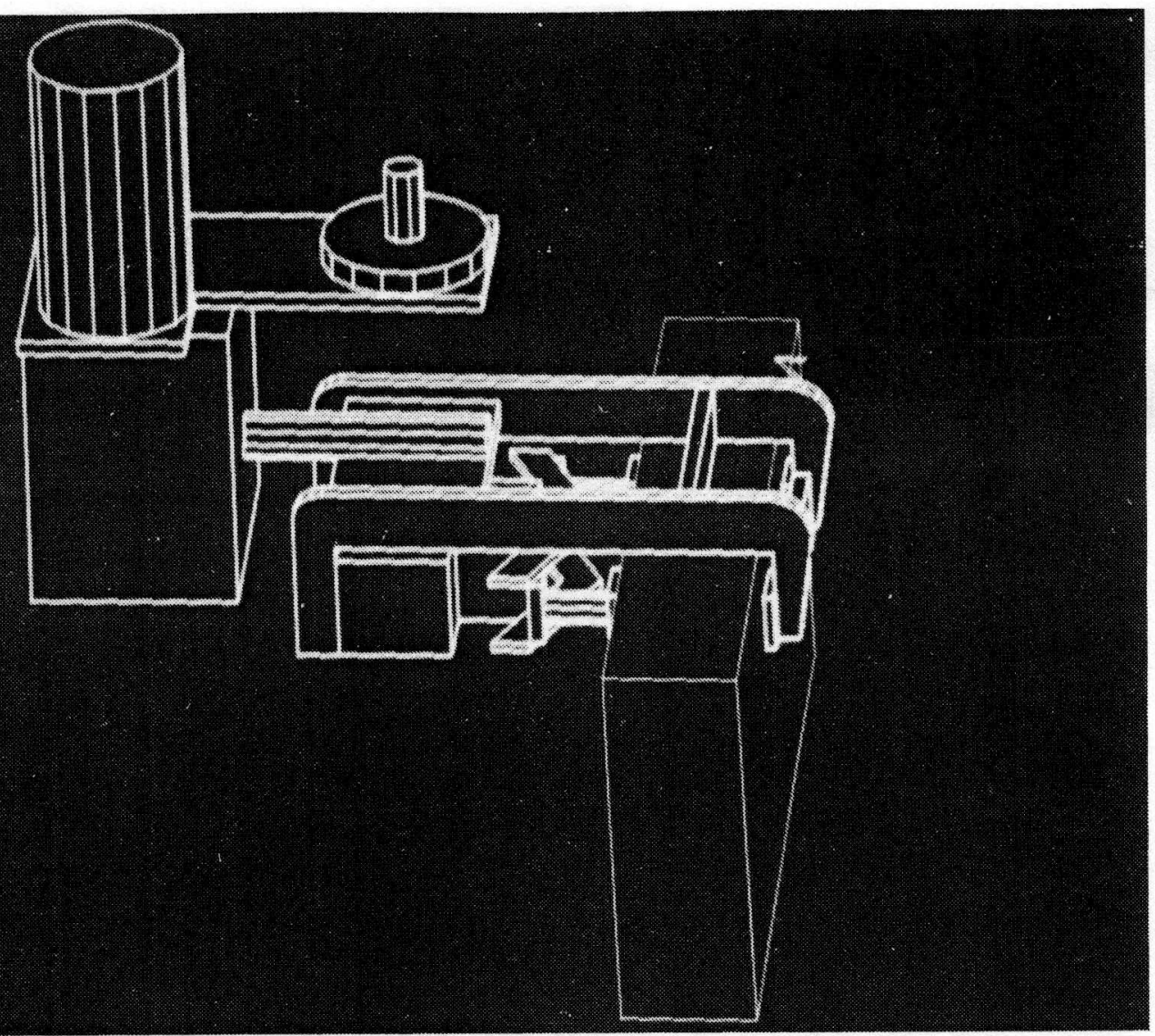

Figure 2. Details of End Effector

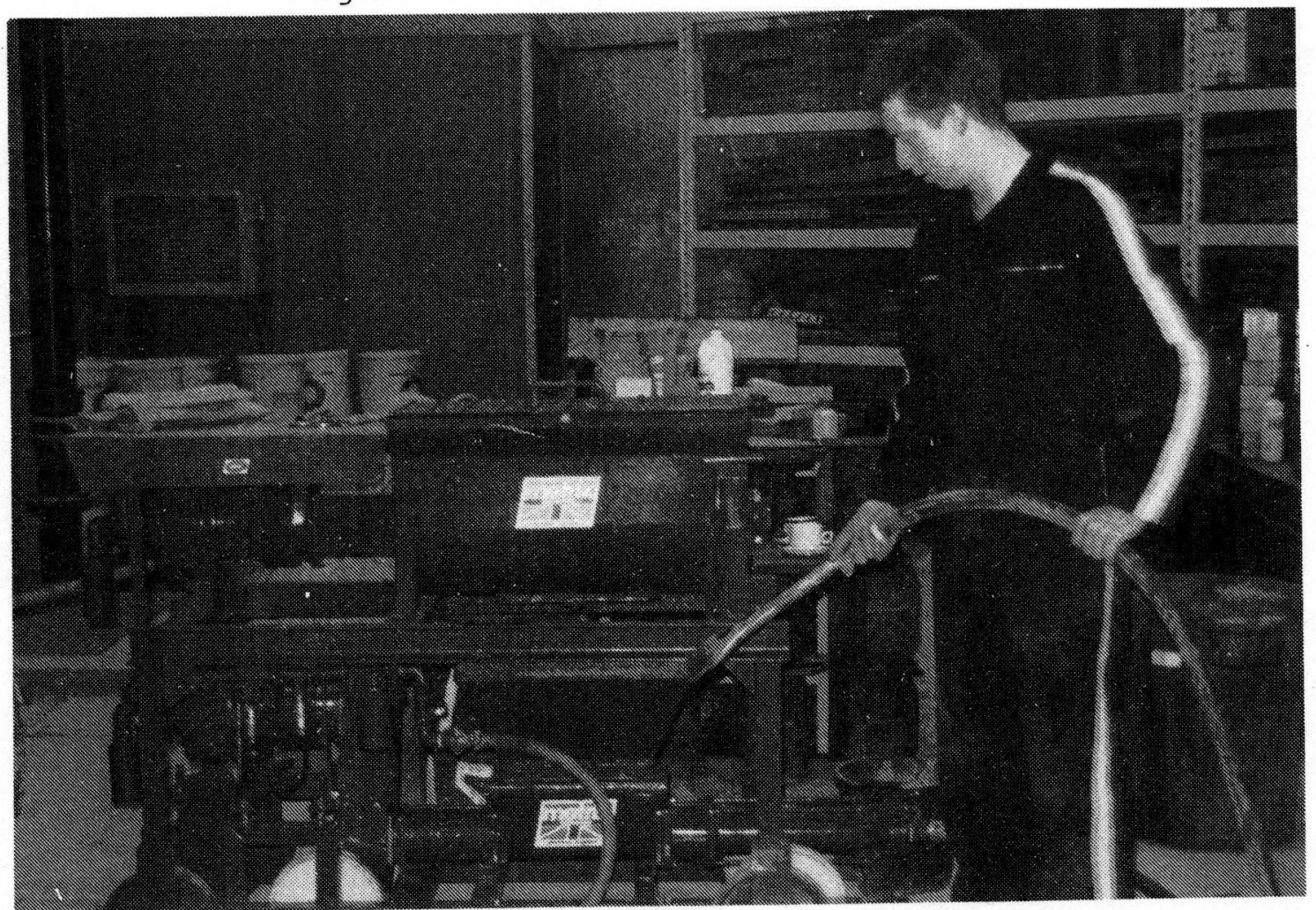

Figure 3. Pump and Delivery System 


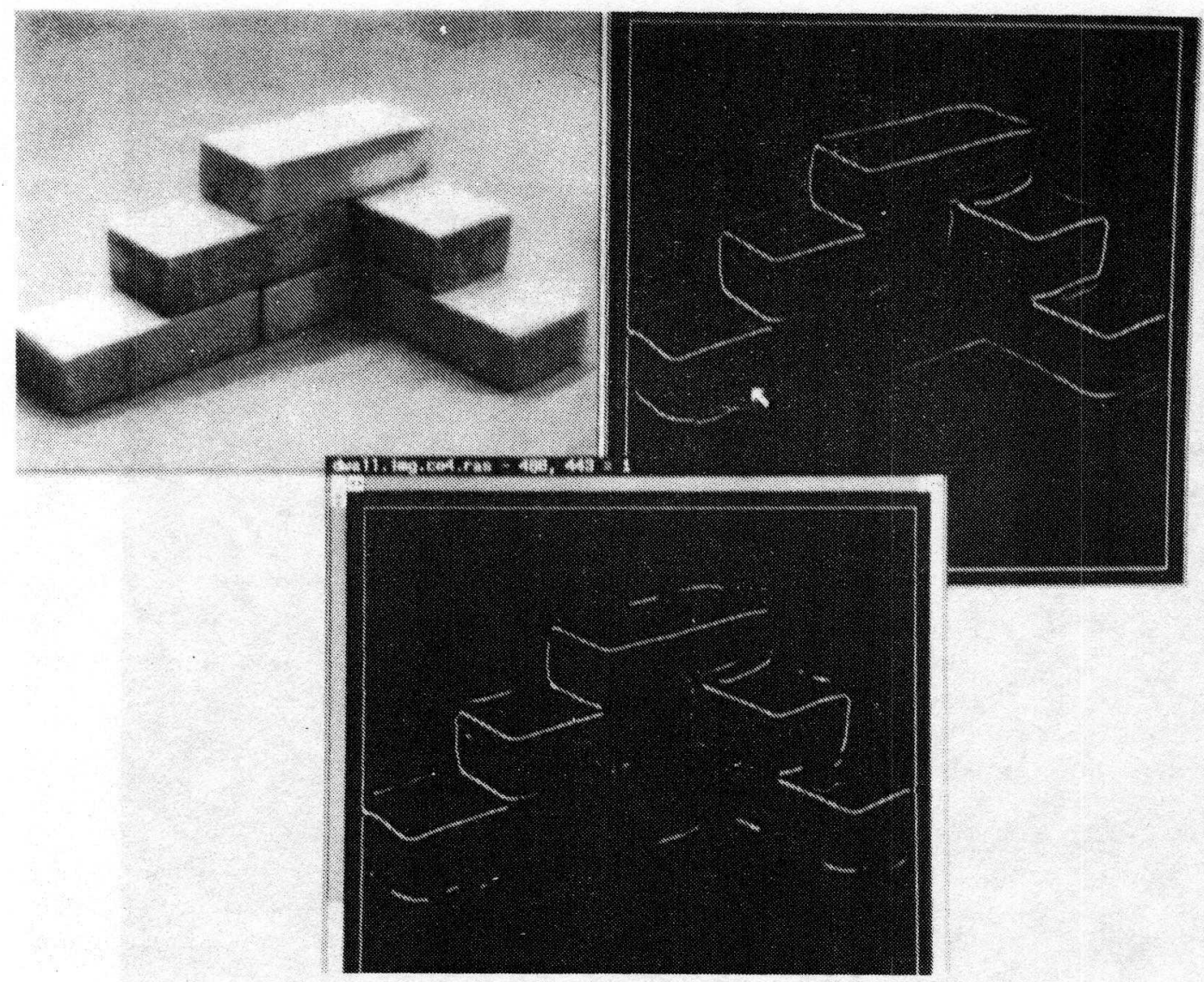

Figure 4. Example of Source and Segmented Wall Scene 